had for 23 years. The four survivors were then in their twentieth, fourth, second, and first postoperative years respectively. The four-year survivor had had pulmonary metastases for three years, controlled by thiotepa. Jones's results compare well with Gordon-Taylor's experience that hindquarter amputation for this tumour in the pelvis produces long-term survival. Though the tumours may reach remarkable size they grow slowly and metastasize late. They are the pre-eminent indication for the operation.

Jones and Gordon-Taylor both had disappointing results when operating upon patients with Paget's disease with sarcoma and with malignant melanoma. However, Jones stated that the operation was justified in the patients with Paget's sarcoma high on the femoral shaft as a palliative measure to overcome the pain of pathological fractures. Of his four patients with malignant melanoma he had only one longterm survivor of 23 years, and his current view is that less radical procedures should be performed.

Osteosarcoma of the femur occurred in three patients in the series. The tumour was sited too proximal for a lower amputation and all three patients had had pathological fractures. All three are alive and well. The longest survivor ( 18 years) was managing a service station and walking 15 miles a day and had worn out four artificial limbs. The appliance given to these patients is usually a Canadian bucket-type of prosthesis; most seem to prefer crutches, which they use with great skill.

\footnotetext{
1 Pringle, J. H., British fournal of Surgery, 1916-17, 4, 283.

2 Gordon-Taylor, G., fournal of the Royal College of Surgeons of Edinburgh, $1959,5,1$.

Gordon-Taylor, G., and Wiles, P., British fournal of Surgery, 1935, 22, 671 .

4 Gordon-Taylor, G., and Patey, D. H., British fournal of Surgery, 1946 34, 61 .

5 Jones, P., Australian and New Zealand fournal of Surgery, 1974, 44, 1.
}

\section{New Targets for Medicine}

Even the most optimistic economists agree that the costs of medical services have recently been growing faster than society can afford. Since the second world war health planners in the western democracies have concentrated their attention on the abolition of financial barriers to medical treatment; but now it seems that new barriers will have to be imposed if their medical services are not to bankrupt these communities.

The serious state of N.H.S. finances sometimes blinds us in Britain to the situation elsewhere in the world. At Oxford last week an international symposium on the provision of medical care (organized by the German Academic Exchange Service) heard speakers from Australia, Canada, Denmark, France, Germany, Norway, Sweden, the U.S.A., and the U.S.S.R. as well as Britain, and despite their very different methods of organizing medical care there was remarkable uniformity in trends and problems.

Much of the rise in the proportion of the national income spent on health in all advanced countries is due to the rapid expansion in medical manpower: every country represented at the conference was training many more doctors, without being at all certain how many more were needed. True, most countries reported that there were some geographical specialties and some medical specialties that were short of doctors; and many besides Britain had to acknowledge their reliance on medical graduates from developing countries. In every country there were many more good applicants than there were places in medical school-and all had found a steady increase in the numbers of women entering medicine. In the U.S.S.R., where selection for medical school is simply conditional on academic standards, $74 \%$ of students are now female. Despite the expansion of training programmes, however, often the current selection procedures for medical students and the type of education they are given still bear little relation to the medical needs of their communities. University medical schools with high academic standards tend to produce graduates who see medicine as a highly technological science practised in large institutions. For the time being there seems no easy solution to the paradox that top quality medical or surgical care uses many more young graduates in the training grades than can ever hope to find permanent appointments in those specialties.

One encouraging trend in both Europe and North America is a rise in popularity of general practice-even in countries where there is no financial incentive for young doctors to choose a career outside hospital. This may be due to better teaching of primary care in medical school, but it seems there is also more interest in the doctor's caring role and some disenchantment with the limited therapeutic success of technological medicine.

At the symposium no-one else was prepared to support a suggestion from Canada that there should be two types of medical schools; some with an emphasis on academic medicine, aimed at producing potential specialists, and others with an admission policy and curriculum intended to produce G.P.s. Much more interest was shown in the use of nurse practitioners in the family health services in Ontario. Perhaps the "medical professional of first contact" should ideally be a doctor; but while doctors remain so expensive to train and to pay in comparison with other health workers there will be strong financial pressures to ration their numbers.

While so great a proportion of the cost of medical care comes from the hospital services, however, it is clearly in those services that fundamental changes of attitude are most needed. In fact there seems to be a growing awareness in all countries that it is no longer possible to hope that every citizen can be offered every medical treatment that is technically practicable. The pace of technological advance has been so fast-and it shows no sign of slowing - that treatment becomes more complex and expensive every day; yet only a few patients who might benefit from such treatment can be offered it, simply because hospitals cannot add to their staff and facilities at a rate equal to the growth of technical knowledge. Given that public attitudes are now such that rationing by cost is no longer acceptable, what answer is there to this dilemma?

One possibility is selection of procedures known to give good results in preference to others that give at best a short-lived relief of symptoms. On this basis, for example, repair of hernia or prolapse would be given priority over many operations for cancer with no real hope of improving the quality of the patient's remaining life. Such a policy is workable when there is little doubt about the response to treatment, but it still leaves a vast grey area where no accurate forecast of outcome is possible. Only when there is better monitoring of the results of different approaches to therapy can a rational selection be made.

A much more radical approach was offered by Professor T. McKeown. Emotionally, the medical profession is still in the era of infectious disease, he argued, when treatment could and did restore patients to normal health. Yet most major health problems in developed countries-cardiovascular disease, arthritis, mental illness-are not curable by modern technology. What is needed, he suggested, is a switch in emphasis to preventive medicine-the identification and removal of environmental hazards and the modification of behaviour known to be dangerous. This approach was supported by $\mathrm{Sir}$ 
George Godber, who suggested that one change in attitude needed was a greater effort to prevent chronicity. Delays in the treatment of reversible conditions were unjustifiable while resources were being diverted to experimental, palliative techniques.

Health education and preventive medicine will need greater emphasis in the future-so much was agreed at the conference. But in discussing national differences in health indices such as maternal and perinatal mortality and life expectancy the delegates agreed that variations in the organization and quality of medical care have only a small effect: almost certainly the most important factors in determining the health of a population are the socioeconomic ones-housing, nutrition, and education. That is why Scandinavia and Switzerland have topped the league for so long and are still drawing away from the rest of the western world.

\section{Forecasting Subarachnoid Haemorrhage}

Second episodes of bleeding after a subarachnoid haemorrhage occur most often in the second and third week after the initial bleed, and the aim of surgical treatment is to forestall these second episodes. Decisions on treatment cannot be taken until the source of the haemorrhage has been identified (unless the possibility of surgery can be ruled out on clinical grounds alone). Though there is still some controversy about the ideal time for carrying out cerebral angiography opinion is crystallizing towards the third or fourth day after haemorrhage, depending on the patient's condition.

If the source of the haemorrhage is shown to be an aneurysm, surgery may prevent further episodes, but the decision depends on factors such as the nature and situation of the aneurysm, the presence of arterial spasm, and the clinical condition. Clearly, then, early recognition is important.

Subarachnoid haemorrhage is still frequently unrecognized-in spite of a signature which is usually quite clear, though sometimes difficult to read and occasionally illegible. Common misdiagnoses include sinusitis, cervical fibrositis, and meningitis. Subarachnoid haemorrhage may have occurred when there is a sudden headache or any cranial symptom of abrupt onset that is followed by vomiting. A first attack of migraine may provide difficulties in differential diagnosis for which the aid of a neurological opinion may be required.

The onset of definitive haemorrhage is frequently unrecognized, so it is not surprising that the forewarnings of haemorrhage which occur in many cases escape detection even more often. These premonitory symptoms may have a very similar character to those of the actual bleed, and often they may represent a small leak from an aneurysm, though this is not always the case. The absence of blood in the cerebrospinal fluid withdrawn by lumbar puncture should not exclude the diagnosis. Even after a frank haemorrhage blood may not appear in the lumbar theca for many hours, and after a symptom that is a forewarning of later haemorrhage it may not be detectable at all. King and $\mathrm{Saba}^{1}$ recently studied the histories of 175 patients in order to determine how many had recognizable warnings which might have led to earlier diagnosis. No fewer than $60 \%$ had complaints which were suffici- ently distinctive to have indicated what was to come. In nearly all there had been a sudden unusual headache, quite distinct from anything that had previously been experienced, severe, and disabling. This was almost equally occipital or frontal, and in a few cases it was retro-orbital. The headache subsided over a few hours or days. Its situation was a poor guide to the location of the aneurysm.

A third of the patients had had recurrent intense headaches of a different nature during the preceding month and $21 \%$ had had them during the preceding six months. This information is only of confirmatory importance, for it is precisely the unique character of the sudden headache which enables it to be distinguished from the many other causes of headache which swamp outpatient clinics and surgeries. However, two thirds of these in whom there was a warning had a history of nausea, and there had been actual vomiting in $19 \%$. Furthermore, just over a third had stiffness of the neck, mostly combined with a suboccipital headache. Overall about $40 \%$ of all cases of haemorrhage which were studied had a history of sudden headache and nausea preceding the main haemorrhage by several days-a combination of symptoms which would have allowed earlier diagnosis, with a consequent opportunity for reducing the mortality. Other symptoms which occurred in the group were syncope or brief coma in $36 \%$, motor or sensory disturbances in $20 \%$, visual disturbances in $12 \%$, and $4 \%$ had seizures.

If the diagnosis is suspected-and the key question is "how did the headache begin ?"-a lumbar puncture should be done after an interval of about 12 hours. If red cells are found in the cerebrospinal fluid or if the supernatant fluid is xanthochromic the patient should be investigated by angiography; but even if this is not so there may be sufficient suspicion of the presence of an aneurysm to warrant investigation. The mortality rate of all subarachnoid haemorrhages is still almost one in three. Any measures which can help to reduce these figures are well worth considering.

King, R. B., and Saba, M. I., New York State fournal of Medicine, 1974, 74, 638.

\section{Explanation Needed}

A recent edition of Pulse prominently published an interview between one of its correspondents and Lord Halsbury, Chairman of the Review Body on Doctors' and Dentists' Remuneration. ${ }^{1}$ Lord Halsbury was reported as commenting on the claim by the profession for an interim pay review (24 August, p. 538). The interview was stated to have taken place "only hours before he [Lord Halsbury] met the J.E.C. [the B.M.A.s Joint Evidence Committee] and the Department of Health in the first round of talks over an immediate $15 \%$ increase in remuneration." The B.M.A. Council discussed this matter in camera at its recent meeting (page 56) and instructed the Secretary to write to Lord Halsbury about his reported remarks. The curious timing of this interview and the alleged subject matter will have startled many doctors and dentists. At the time the B.M.F. went to press Lord Halsbury had made made no official public statement about the incident. The two professions will welcome a prompt statement from the Chairman of the Review Body or their present unease may rapidly develop into doubt about his impartiality as a chairman.

\footnotetext{
${ }^{1}$ Pulse, 21 September, 1974, p. 1.
} 